https://doi.org/10.15407/ujpe64.7.635

\author{
M. SCHEVER ${ }^{1,2}$ \\ for the JUNO Collaboration \\ ${ }^{1}$ Institut für Kernphysik, Forschungszentrum Jülich \\ (52425 Jülich, Germany; e-mail: m.schever@fz-juelich.de) \\ 2 III. Physikalisches Institut B, RWTH Aachen University \\ (52074 Aachen, Germany)
}

\title{
STATUS OF THE JIANGMEN UNDERGROUND NEUTRINO OBSERVATORY
}

\begin{abstract}
The Jiangmen Underground Neutrino Observatory (JUNO) is a next generation multipurpose antineutrino detector currently under construction in Jiangmen, China. The central detector, containing 20 kton of a liquid scintillator, will be equipped with $\sim 1800020$ inch and 25600 3 inch photomultiplier tubes. Measuring the reactor antineutrinos of two powerplants at a baseline of $53 \mathrm{~km}$ with an unprecedented energy resolution of $3 \% / \sqrt{E(\mathrm{MeV})}$, the main physics goal is to determine the neutrino mass hierarchy within six years of run time with a significance of 3-4 $\sigma$. Additional physics goals are the measurement of solar neutrinos, geoneutrinos, supernova burst neutrinos, the diffuse supernova neutrino background, and the oscillation parameters $\sin ^{2} \theta_{12}, \Delta m_{12}^{2}$, and $\left|\Delta m_{\text {ee }}^{2}\right|$ with a precision $<1 \%$, as well as the search for proton decays. The construction is expected to be completed in 2021.
\end{abstract}

Ke ywords: antineutrino detector, reactor antineutrinos, supernova neutrinos, proton decay, neutrino mass hierarchy.

\section{Introduction}

The Jiangmen Underground Neutrino Observatory (JUNO) is a 20 kton liquid scintillator (LS) detector currently under construction in the south of China close to Jiangmen. The LS is contained in a $35.4 \mathrm{~m}$ diameter acrylic sphere and monitored by $\sim 18000$ 20 inch photomultiplier tubes (PMTs), allowing for an unprecedented energy resolution of $3 \% / \sqrt{E(\mathrm{MeV})}$. A complementary system of 256003 inch PMTs facilitates to use the concept of double calorimetry[1]. In order to reduce the external background and to track and veto cosmogenic muons, the Central Detector (CD) is submerged in a cylindrical water Cherenkov detector filled with ultra-pure water. The main goal of JUNO is to determine the neutrino mass hierarchy $(\mathrm{MH})$ by measuring the oscillations of reactor antineutrinos emitted by two powerplants, Taishan and Yiangjian, with a final thermal power of $35.8 \mathrm{GW}_{\text {th }}$ at a baseline of $53 \mathrm{~km}$. Furthermore, measurements of the oscillation parameters $\sin ^{2} \theta_{12}, \Delta m_{12}^{2}$, and $\left|\Delta m_{e e}^{2}\right|$ can be achieved with subpercentage precision. $\mathrm{Nu}$ merous additional physics goals exist [2], of which

(C) M. SCHEVER, 2019

ISSN 2071-0194. Ukr. J. Phys. 2019. Vol. 64, No. 7 measuring solar neutrinos and geoneutrinos are reviewed in the following. The Taishan Antineutrino Observatory (TAO) will be built and operated next to the Taishan power plant to reduce systematic effects in the reactor antineutrino spectrum measured by JUNO.

\section{Neutrino Physics Programme at JUNO}

\subsection{Reactor Antineutrinos}

To reach the primary goal of determining the neutrino mass hierarchy, JUNO aims at the detection of reactor antineutrinos based on the Inverse Beta Decay (IBD) of protons occuring in the LS in the CD, where the $e$-flavor antineutrino reacts with a proton producing a positron and a neutron according to

$\bar{\nu}_{e}+p \rightarrow e^{+}+n$.

The IBD signature is the coincidence of a prompt and a delayed signal. The prompt signal stems from the energy loss and the subsequent annihilation of a positron taking place effectively instantaneously after its creation. Since the mass of a neutron is much larger than the mass of a positron, the energy of the 


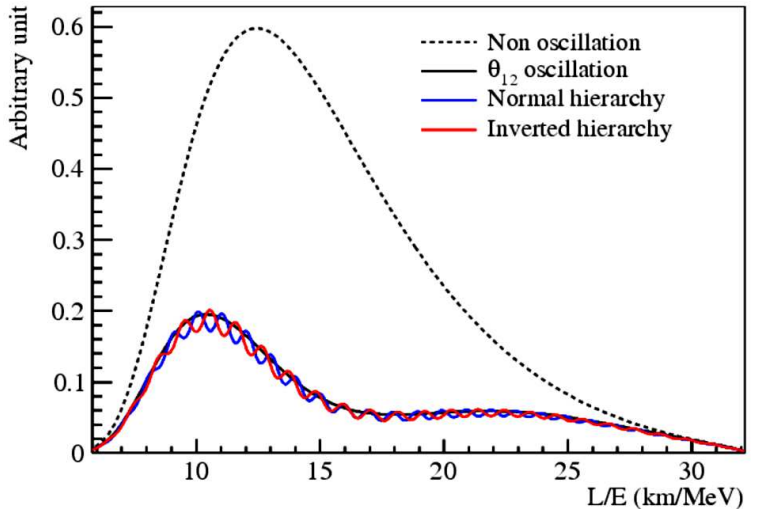

Fig. 1. Unoscillated reactor neutrino flux (dotted line) and the relative shape differences for the NH and the IH. From [2]

positron relates to the energy of an antineutrino. The delayed signal stems from the neutron being captured by hydrogen in the LS with a mean time of $\sim 200 \mu$ s after undergoing the thermalization. The neutron capture on protons emits a photon with an energy of $2.2 \mathrm{MeV}$.

The time coincidence of both the prompt and delayed signals, together with their vertex positions and the energy constraint, allows for the IBD signal detection and the background rejection. Several sources have to be considered for the background. The dominant background source is the cosmogenic isotopes ${ }^{9} \mathrm{Li}$ and ${ }^{8} \mathrm{He}$, which are produced through the spallation by cosmogenic muons traversing through the detector. Their $\left(\beta^{-}+n\right)$-decay channel mimics the coincidence of the prompt and delayed signals of the IBD signature. Featuring a life time of $256 \mathrm{~ms}$ and

Expected signal and background rates per day with various selection cuts. From [2]

\begin{tabular}{|c|c|c|c|c|c|c|}
\hline Section & IBD & Geo- $\nu \mathrm{s}$ & Accidental & ${ }^{9} \mathrm{Li} /{ }^{8} \mathrm{He}$ & Fast $n$ & $(\alpha, n)$ \\
\hline- & 83 & 1.5 & $\sim 5.7 \times 10^{4}$ & 84 & - & - \\
\hline $\begin{array}{l}\text { Fiducial } \\
\text { volume }\end{array}$ & 76 & 1.4 & \multirow{3}{*}{410} & 77 & \multirow{5}{*}{0.1} & \multirow{5}{*}{0.5} \\
\hline Energy cut & \multirow{3}{*}{73} & \multirow{3}{*}{1.3} & & \multirow{3}{*}{71} & & \\
\hline Time cut & & & & & & \\
\hline Vertex cut & & & 1.1 & & & \\
\hline Muon Veto & 60 & 1.1 & 0.9 & 1.6 & & \\
\hline Combined & 60 & & 3.8 & & & \\
\hline
\end{tabular}

$172 \mathrm{~ms}$, respectively, $99 \%$ of the ${ }^{9} \mathrm{Li}$ and ${ }^{8} \mathrm{He}$ isotopes produce IBD-like signatures at a $3 \mathrm{~m}$ distance to the muon track $[3,4]$. Therefore, the strategy for the muon veto includes a partial volume veto of the area of the liquid scintillator contained in a cylinder with a radius of $3 \mathrm{~m}$ around the muon track for a time of $1.2 \mathrm{~s}[5]$. Other considerable background sources are the following:

- Accidental coincidences by natural radioactivity, mostly from the surrounding rock and the PMT glass.

- Fast neutrons produced by cosmogenic muons which travel through the surrounding rock or through the water at the corner of the detector. They can mimic the IBD signature by scattering off a proton and undergoing the subsequent neutron capture in the LS.

- Geoneutrinos produced in the radioactive decays of $\mathrm{U}$ and $\mathrm{Th}$ from inside the Earth. Causing the same signal like the reactor antineutrinos, their contribution to the reactor antineutrino energy spectrum is handled through the known $\beta$-decay spectra of $\mathrm{U}$ and Th.

- $(\alpha, n)$-background originating from the $\alpha$ particles of the $\mathrm{U}$ and $\mathrm{Th}$ chain and reacting with ${ }^{13} \mathrm{C}$ in the LS and producing a neutron and ${ }^{16} \mathrm{O}$. The IBD coincidence signature can be mimicked in the case where a neutron is fast enough or ${ }^{16} \mathrm{O}$ emits a photon during the deexcitation.

The expected rates of both the IBD reactor neutrino signal and the above-mentioned backgrounds are summarized in Table.

The neutrino $\mathrm{MH}$ is determined by relating the measured reactor antineutrino spectrum to the $\mathrm{MH}-$ dependent survival probabilities for antielectron neutrinos conditioned by neutrino oscillations. Here, the measured reactor antineutrino energy spectrum is represented by the prompt energy spectrum of the positrons produced in the IBDs. The antielectron neutrino survival probability is given by

$$
\begin{aligned}
& P_{\bar{\nu}_{e} \rightarrow \bar{\nu}_{e}}=1-\sin ^{2} 2 \theta_{13}\left(\sin ^{2} \theta_{12} \sin ^{2} \Delta_{32}+\right. \\
& \left.+\cos ^{2} \theta_{12} \sin ^{2} \Delta_{13}\right)-\sin ^{2} 2 \theta_{12} \cos ^{4} \theta_{13} \sin ^{2} \Delta_{12}
\end{aligned}
$$

with $\Delta_{i j}=\left(\Delta m_{i j}^{2} L\right) /(4 E)$ and shown for both cases of the normal hierarchy $(\mathrm{NH})$ and the inverted hierarchy $(\mathrm{IH})$ in Fig. 1.

The $\chi^{2}$-based analysis of the reactor antineutrino energy spectrum determines the neutrino mass hierarchy with a sensitivity $\Delta \chi^{2}$ by fitting the energy spectrum with the expected spectra both for the $\mathrm{NH}$ 
and IH. The obtained sensitivity depends both on the acquired amount of statistics and the energy resolution. Figure 2 shows the $\Delta \chi^{2}$-contour plot for different sensitivity levels depending on a variable energy resolution and a range of the number of IBD events included in the analysis. The latter is normalized to the expected number of events after 6 years of the data acquisition with $35.8 \mathrm{GW}_{\text {th }}$ reactor power, corresponding to 100,000 IBD events. With this amount of detected events and the design energy resolution of $3 \% / \sqrt{E(\mathrm{MeV})}$, the reachable sensitivity is expected to be $3-4 \sigma$, corresponding to $9-16 \Delta \chi^{2}$.

Additionally, JUNO will be able to improve the precision of the oscillation parameters $\sin ^{2} \theta_{12}, \Delta m_{12}^{2}$, and $\left|\Delta m_{e e}^{2}\right|$ to the subpercentage level of $0.67 \%$, $0.50 \%$, and, $0.44 \%$, respectively.

\subsection{Solar neutrinos}

The Sun is a powerful source of electron neutrinos. The neutrinos are produced in the nuclear fusion reactions and emitted with the energy of $\mathcal{O}(1 \mathrm{MeV})$. Their study yields the possibility to gain knowledge in the context of neutrino properties (e.g., the Mikheyev-Smirnov-Wolfenstein (MSW) effect [6]) as well as the Sun (e.g. the solar metallicity problem [7]).

The JUNO experiment is principally well suited for the detection of solar neutrinos via the electron scattering due to the low energy detection threshold, the high energy resolution, the high radiopurity, and the large mass. The focus lies on the neutrinos emitted from the ${ }^{8} \mathrm{~B}$ and ${ }^{7} \mathrm{Be}$ chains.

Since a single energy deposition of the scattering electron is the event signature, the resulting experimental challenge is the rejection of the enormous background. Dominant background sources are natural radioactivity $\left({ }^{210} \mathrm{Po},{ }^{210} \mathrm{Bi},{ }^{14} \mathrm{C}\right.$ and its pile-up, ${ }^{85} \mathrm{Kr}$, and the ${ }^{238} \mathrm{U}$ - and ${ }^{232} \mathrm{Th}$-chains [8]) and the cosmogenic isotopes ${ }^{10} \mathrm{C}$ and ${ }^{11} \mathrm{C}$. The expected detection rates are $\sim 10^{4}$ events per day for ${ }^{7}$ Be and $\sim 90$ events per day for ${ }^{8} \mathrm{~B}$.

\subsection{Geoneutrinos}

While the Earth's surface heat flow has been measured to be $(46 \pm 3) \mathrm{TW}$ [9], the contribution of the radiogenic heat in contrast to the primordial heat remains unclear till now. Therefore, knowledge of the absolute abundance of $U$ and $T h$ in the Earth is required. Their abundance is accessible through the antielectron neutrino flux caused by the radioactive $\beta$-decays from the ${ }^{238} \mathrm{U}$ and ${ }^{232} \mathrm{Th}$ chains.

ISSN 2071-0194. Ukr. J. Phys. 2019. Vol. 64, No. 7

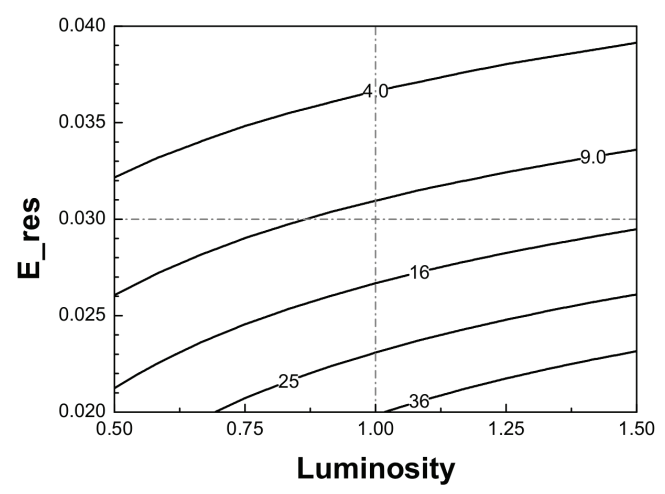

Fig. 2. Iso- $\Delta \chi^{2}$ contour plot as a function of the acquired statistics labeled as the luminosity and the energy resolution. The nominal luminosity marked by the vertical dotted line corresponds to a run time of 6 years and 100,000 IBD events. The design energy resolution is marked by the horizontal dotted line. From [2]

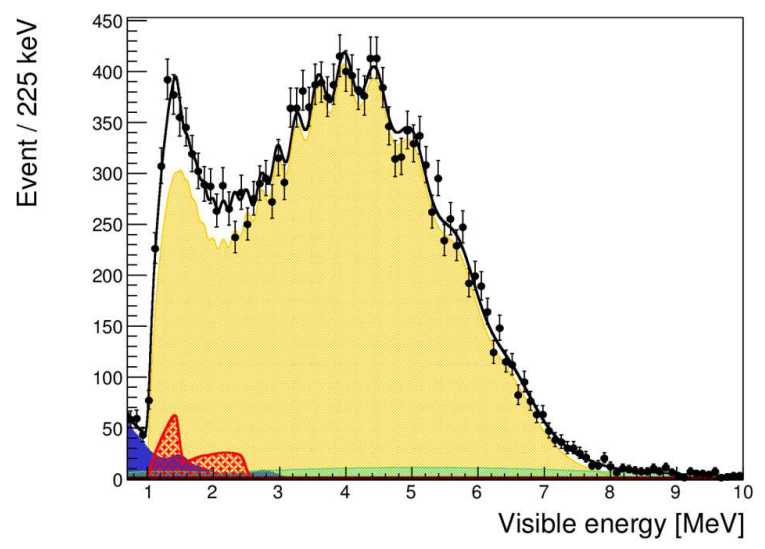

Fig. 3. Expected geoneutrino signal (grid shade) and background spectra for one year of measurement. Background contributions are the reactor antineutrinos (light grey), the cosmogenic isotopes ${ }^{9} \mathrm{Li}$ and ${ }^{8} \mathrm{He}$ (flat light grey), the accidental background (dark grey), and the $(\alpha, n)$-background (small). The total sum for the best fit is indicated by the black line. From [2]

JUNO aims at measuring these so-called geoneutrinos expected with a rate of 400 events per year [2]. This would yield the world's largest sample of geoneutrinos in less than one year. The experimental challenge is the large background of reactor antineutrinos which can only be handled by the subtraction of both spectra. Further background sources are the cosmogenic isotopes ${ }^{9} \mathrm{Li}$ and ${ }^{8} \mathrm{He}$, accidental background, and $(\alpha, n)$-background. Figure 3 demonstrates the expected geoneutrino signal and background spetra for one year of measurements. 


\section{The JUNO Experiment}

The JUNO experiment is located close to Jiangmen in China, at a distance of $53 \mathrm{~km}$ to both the Taishan and Yiangjian powerplants. The detector is built underground with an overburden of $700 \mathrm{~m}$ of a granite rock to reduce the amount of cosmogenic muons as the background source.

This section describes the following subsystems of the JUNO detector: the Central Detector (CD), the Water Pool (WP), the Top Tracker (TT), the Calibration System, activities and apparatus in the context of the LS purification, and the Taishan Antineutrino Observatory (TAO).

\section{The central detector}

The CD is an acrylic sphere with a diameter of $35.4 \mathrm{~m}$ containing $20 \mathrm{kton}$ of LS. It is equipped with 1800020 inch Photo-Multiplier Tubes (PMTs) and 256003 inch PMTs. The PMTs are mounted on a stainless steel truss surrounding the acrylic sphere with a distance of $1.8 \mathrm{~m}$. The resulting photocoverage is $78 \%$. A high quantum efficiency of $\sim 30 \%$ is required in order to reach the unprecedented design energy resolution of $3 \% / \sqrt{E(\mathrm{MeV})}$. Out of the 18000 20 inch PMTs, 5000 are dynode PMTs produced by Hamamatsu Photonics K.K., the remaining PMTs are Micro Channel Plate PMTs and manufactured by the Chinese company North Night Vision Technology Co. Ltd. Shielding against the Earth's magnetic field is ensured by the compensation through electromagnetic coils.

\section{The water pool and the top tracker}

The CD is submerged into the cylindrically shaped WP containing $40 \mathrm{kton}$ of ultra-pure water to provide shielding from the radioactivity of the surrounding rock and the PMT glass. The WP has a diameter of $43.5 \mathrm{~m}$ and is equipped with 240020 inch PMTs to detect the Cherenkov light of muons traversing the JUNO detector. The TT is placed on the WP top. It was a part of the former Opera detector [10] and consists of three layers of a plastic scintillator with a spatial resolution of $2.6 \times 2.6 \mathrm{~cm}^{2}$ and a coverage of approximately $60 \%$ of the surface of the top of the WP. Together, the WP and the TT enable one to track cosmogenic muons providing the foundation for a partial volume muon veto.

\section{8}

\section{Calibration}

In order to achieve an energy scale uncertainty of less than $1 \%$, the efficient calibration is of great importance. Four calibration systems are planned to be implemented to provide the basis for a thourough calibration. The first calibration system is the $\mathrm{Au}$ tomated Calibration Unit (ACU) which can be operated 1-dimensionally along the vertical axis in the center of the detector. The second calibration system is the Cable Loop System (CLS), and the third is the Guide Tube Calibration System (GTCS). Both CLS and GTCS can be operated 2-dimensionally, the first in a fixed vertical plane and the latter along a fixed longitude of the CD bound to a guide tube. The fourth calibration system, the Remotely Operated Vehicle (ROV), is steerable in all 3 dimensions and can move freely within the LS in the CD. Furthermore, the double calorimetry system including both the 20 inch and 3 inch PMTs provides an additional calibration strategy, especially with respect to the systematics of the large PMTs due to a multiplicity in the photo-electron detection.

\section{LS purification}

In order to prepare the mixing of the LS components and the online purification procedure for the filling of the JUNO CD, as well as to gain experience in the system cleanliness and leak-tightness, distillation, and stripping, pilot plants are currently tested at the Daya Bay Neutrino Laboratory [11].

The LS purification aims at decreasing the amount of radioimpurities primary due to ${ }^{238} \mathrm{U},{ }^{232} \mathrm{Th}$, and ${ }^{40} \mathrm{~K}$. For ${ }^{238} \mathrm{U}$ and ${ }^{232} \mathrm{Th}$, the abundances in the range of $10^{-15}-10^{-17} \mathrm{~g} / \mathrm{g}$ are targeted. Simultaneously, the attenuation length in the wavelength interval 350$550 \mathrm{~nm}$ is improved to exceed $25 \mathrm{~m}$ for a wavelength of $430 \mathrm{~nm}$. The gas stripping of the LS with steam and nitrogen extracts radioactive gases, in particular ${ }^{85} \mathrm{Kr},{ }^{39} \mathrm{Ar}$, and ${ }^{222} \mathrm{Rn}$.

\section{The Taishan Antineutrino Observatory}

In order to determine the neutrino $\mathrm{MH}$ from the oscillated reactor antineutrino spectrum measured by the JUNO detector, a precise knowledge of the unoscillated spectrum is required. The existing model for the energy-dependent reactor flux is subject, however, to both the anomalous bump observed in reactor antineutrino spectra at $5 \mathrm{MeV}$ and a fine structure yet unknown [12]. Therefore, TAO will be placed

ISSN 2071-0194. Ukr. J. Phys. 2019. Vol. 64, No. 7 
in $30 \mathrm{~m}$ distance to a $4.6 \mathrm{GW}_{\mathrm{th}}$-power core of the Taishan powerplant to measure the shape of the unoscillated reactor antineutrino reference spectrum for JUNO. The spherical detector will be filled with several tons of $\mathrm{Gd}-\mathrm{LS}$ to detect the antineutrinos via the IBD reaction. Being equipped with Silicon Photomultipliers (SiPM) featuring a photo-electron (PE) detection efficiency of $\sim 50 \%$ at the full coverage, a light yield of $4500 \mathrm{PEs}$ at an energy of $1 \mathrm{MeV}$ will be reached, resulting in an energy resolution better than $3 \% \sqrt{E(\mathrm{MeV})}$. The Gd-LS will be operated at -50 degree Celsius to reduce the SiPM noise [13].

\section{Conclusion}

JUNO is a 20 kton liquid scintillator detector currently under construction in the south of China, close to Jiangmen. The physics main goal is to determine the neutrino $\mathrm{MH}$ based on the detection of reactor antineutrinos at a baseline of $53 \mathrm{~km}$ reaching 3-4 $\sigma$ significance after 6 years of data taking with $35.8 \mathrm{GW}_{\mathrm{th}}$ reactor power. Therefore, an unprecedented energy resolution of $3 \% / \sqrt{E(\mathrm{MeV})}$ based on a light yield of $1200 \mathrm{PE} / \mathrm{MeV}$ and an energy scale uncertainty $<1 \%$ is required. Furthermore, the physics programme is extended to the detection of terrestrial and astrophysical neutrinos. The oscillation parameters $\sin ^{2} \theta_{12}$, $\Delta m_{12}^{2}$, and $\left|\Delta m_{e e}^{2}\right|$ will be measured at a subpercentage precision level. The construction is expected to be completed in 2021 .

1. Miao He on behalf of the JUNO Collaboration. Double calorimetry system in JUNO. arXiv:1706.08761 (2017).

2. F. An et al., (JUNO Collaboration). Neutrino physics with JUNO. arXiv:1507.05613 (2016).

3. M. Grassi, J. Evslin, E. Ciuffoli, X. Zhang. Showering cosmogenic muons in a large liquid scintillator. JHEP $\mathbf{0 4 9}$, 09 (2014).

4. M. Grassi, J. Evslin, E. Ciuffoli, X. Zhang. Vetoing cosmogenic muons in a large liquid scintillator. arXiv:1505.05609 (2015).

5. C. Genster, M. Schever, L. Ludhova, M. Soiron, A. Stah1, C. Wiebusch. Muon reconstruction with a geometrical model in JUNO. arXiv:1906.01912 (2018).
6. A.Yu. Smirnov. The MSW effect and solar neutrinos. arXiv:0305106 (2003).

7. A. Serenelli, S. Basu, J. W. Ferguson, M. Asplund. New solar composition: The problem with solar models revisited. Astrophys. J. 705, L123 (2009).

8. M. Agostini et al., (Borexino Collaboration). Comprehensive measurement of pp-chain solar neutrinos. Nature $\mathbf{5 6 2}$, 496 (2018).

9. C. Jaupart, S. Labrosse, J.C. Mareschal. Temperatures, heat and energy in the mantle of the Earth. In Treatise on Geophysics, vol. 7: Mantle dynamics, Eds. by D. Bercovici, G. Schubert (Elsevier, 2007).

10. T. Adam et. al. The OPERA experiment Target Tracker. Nucl. Inst. \& Meth. in Phys. Res. A 577 (3), 523 (2007).

11. P. Lombardi et. al. Distillation and stripping pilot plants for the JUNO neutrino detector: Design, operations and reliability. arXiv:1902.05288 (2019).

12. D.V. Forero, R. Hawkins, P. Huber. The benefits of a near detector for JUNO. arXiv:1710.07378 (2017).

13. Jun Cao (JUNO Collaboration). Measuring High Resolution Reactor Neutrino Spectrum with JUNO-TAO. Talk presented at the Technical Meeting on Nuclear Data for Anti-neutrino Spectra and Their Applications, IAEA, Vienna, Apr. 23-26 (2019).

Received 15.08.19

\section{М. Шевер, від імені Колаборацій JUNO}

\section{СТАН ПІДЗЕМНОї}

\section{ОБСЕРВАТОРЇ̈ НЕЙТРИНО В ЖІАНГМЕНІ}

$\mathrm{P}$ е $з$ ю м е

Підземна Обсерваторія Нейтрино в Жіангмені (JUNO) є багатоцільовим детектором антинейтрино нового покоління, що споруджується в Китаї. Центральний детектор, що містить 20 кілотон рідинного сцинтилятора, буде оснащено трубками фотопомножувачів, 17571 штук по 20 дюймів та 25600 по 3 дюйми. У процесі вимірювання антинейтрино від двох реакторів з базою 53 км при безпрецедентній роздільній здатності по енергії $3 \% / \sqrt{E}$ МеВ основною метою є визначення впродовж шести років роботи ієрархії мас нейтрино 3 точністю 3-4 $\sigma$. Додатковими фізичними цілями $€$ вимірювання сонячних нейтрино, геонейтрино, нейтрино від вибуху супернової, нейтринного фону дифузної супернової, параметрів осциляції $\sin ^{2} \theta_{12}, \Delta m_{12}^{2},\left|\Delta m_{e e}^{2}\right|$ з точністю $<1 \%$, а також пошуки розпаду протона. Планується закінчити конструкцію у 2021 році. 